\title{
Preparation and Thermal and Anti-UV Properties of Chitosan/Mica Copolymer
}

\author{
Yih-Sheng Huang, Sheng-Haur Yu, Yea-Ru Sheu, and Kuo-Shien Huang \\ Department of Polymer Materials, Kun Shan University, Yung Kang, Tainan 71003, Taiwan \\ Correspondence should be addressed to Kuo-Shien Huang, ksusec@mail.ksu.edu.tw
}

Received 30 July 2010; Accepted 17 November 2010

Academic Editor: Kin Tak Lau

Copyright () 2010 Yih-Sheng Huang et al. This is an open access article distributed under the Creative Commons Attribution License, which permits unrestricted use, distribution, and reproduction in any medium, provided the original work is properly cited.

\begin{abstract}
This experiment aims to produce a free radical while annoying the oxidizing-reducing reagent of the ammonium persulfate and the sodium bisulfite under nitrogen, then trigger copolymerization between modified-mica and chitosan to prepare a variety of copolymers. This experiment also aims to study the related properties of these copolymer materials. The experimental data shows that the copolymer has more thermal stability and better absorption of UV than chitosan. But the above physical properties will be less if the mica ratio in copolymer is more than $8 \%$. On the other hand, the SEM photo of the microstructure also shows that the modified mica distributes homogeneously on the surface of the film of the copolymer.
\end{abstract}

\section{Introduction}

Chitin and its deacetylated product, chitosan, are the most abundant biological resources in nature, second only to plant fiber. They widely exist in the shells of arthropods such as shrimps, crabs, and insects. Chitosan, also called polyaminoglucose or poly $(\beta$ - $(1,4)-2$-amino-2-deoxyD-glucose, is an abundant polysaccharide that is derived by deacetylation of chitin, a carbohydrate composed of $N$ acetylated glucosamine monomers with a $\beta-1,4$ bond [14]. Chitosan has unique properties, such as biocompatibility and biodegradability (Ravi Kumar, 2000 [5]; Ravi Kumar, 2004 [6]; Felt et al., 1998 [7]; Hirano et al., 1989 [8]). Its degradation products are nontoxic, noncarcinogenic, and antibacterial (Muzzarelli, 1997 [9], Felt et al., 1998 [7]; Bersch et al., 1995 [10]). Therefore, chitosan-derived films, fibers, and nanoparticles have been widely studied and developed in fields such as biomedical materials, wastewater treatment, textiles, and biotechnology [11]. Because of its molecular structure, chitosan is fragile with poor toughness and thermal properties. It can be made into derivative products with better mechanical properties by altering the drying procedure, physical mixing, or chemical grafting. These improvements enhance chitosan's applicability $[12,13]$.
Chitosan has three types of active groups, which are $\mathrm{C}_{2}-\mathrm{NH}_{2}, \mathrm{C}_{3}-\mathrm{OH}$, and $\mathrm{C}_{6}-\mathrm{OH} . \mathrm{C}_{2}-\mathrm{NH}_{2}$ is a primary amino group with a lone pair of electrons; as such, its nucleophilicity is very strong. $\mathrm{C}_{3}-\mathrm{OH}$ is a secondary hydroxyl group that cannot rotate freely due to high steric hindrance; its activity is poor. $\mathrm{C}_{6}-\mathrm{OH}$ is a primary hydroxyl group capable of free rotation in space due to low hindrance; its ability to rotate imparts this moiety with a higher activity. These active groups can be utilized in multiple physical or chemical modifications $[4,14]$. Chitosan modification by graft copolymerization is one of the recent research objectives. This field of study can be divided into two types: (1) vinyl graft polymerization and (2) nonvinyl graft polymerization. With the goal of altering the charge distribution of chitosan, group 1 consists of chemically-initiated vinyl graft polymerization and irradiated vinyl graft polymerization. Group 2, which aims to change the biocompatibility of chitosan, includes graft copolymerization and ring-opening graft copolymerization [15].

Compared with zero- or one-dimensional nanomaterials, lamella or layered nanomica can impart more significant improvements to the mechanical strength of a polymer. Nanomica displays a gas barrier, flame retardancy, high thermal conductivity, abrasion resistance, UV resistance, 
and high strength. The melting point of nanomica is as high as $800^{\circ} \mathrm{C}$. Nanomica is a typical two-dimensional reinforced filler, which can enhance toughness and crack resistance of copolymer materials. Mica's polarization effect and interlayer light interference effect allow it to have UV shielding properties [16-19].

Polymer/Layered Silicate (PLS) is an emerging copolymer material. As compared with standard polymer copolymer materials, PLS displays several beneficial qualities. PLS requires little filler, as only a small amount can allow the copolymer material to have excellent strength, elasticity modulus, toughness, and barrier performance. Also, this copolymer has excellent thermal stability and geometry stability. While oriented in two dimensions, silicate platelets can reinforce the planar film, allowing high barrier properties. PLS nanocopolymers are lighter than conventional polymer fillers, with a small amount being sufficient to greatly lower the cost. Additionally, PLS is flame retardant and thermally resistant. This phenomenon is due to a barrier to heat diffusion and mass transmission in combustion caused by the lamellar silicate platelet restriction of polymer chains.

Preparation of organic/inorganic copolymer materials has been widely studied and applied to improved performance in electrical, optic, and biomedical fields. However, common problems exist, such as irregular inorganic phase distribution, inorganic morphology control, and interfacial dispersion methods. In this study, modified mica was copolymerized with a green material, chitosan, in order to obtain a chitosan/mica copolymer material. The material could thus be used to improve the thermal and mechanical properties of chitosan and to prepare high performance and multifunctional copolymer material.

\section{Experimental}

2.1. Reagents. This study involved the use of chitosan (CS, degree of deacetylation $85 \%$, reagent class, Taiwan Kaohsiung Applied Chemistry Co., Ltd.), modified nanomica (NM-967, industrial class, with vinyl terminal group, Taiwan Chengyi Chemical Plant Co., Ltd.), and nonionic surfactant (ES-100, DOW Chemicals). Monosodium disulfite (MSDS), ammonium persulfate (APS), and acetic acid were purchased from Toku Medical Co., Ltd., Japan.

2.2. Experimental Procedure. Dissolve $2 \mathrm{~g}$ CS in $60 \mathrm{~mL} 2 \%$ aqueous acetic acid solution, place it into a thermostatic water recycling tank, fill it with nitrogen gas, and stir while refluxing at $30^{\circ} \mathrm{C}$. After $5 \mathrm{~min}$, add $0.5 \mathrm{~mL}$ of $10 \%$ nonionic surfactant. Disperse an appropriate amount of mica in $2 \%$ aqueous acetic acid solution, stir for $5 \mathrm{~min}$ at room temperature, and add $0.5 \mathrm{~mL}$ of $10 \%$ nonionic surfactant. After $15 \mathrm{~min}$, pour the solution from step 2 into the solution from step 1 and add $0.1 \mathrm{~g}$ APS and $0.1 \mathrm{~g}$ MSDS, separately. Stir for $4 \mathrm{~h}$ in a thermostatic water recycling tank at $30^{\circ} \mathrm{C}$. Using a rapid defoaming agitator, remove the foams in the prepared chitosan/mica emulsion. Take a certain amount of copolymer emulsion and put it in a petri dish to bake for $20 \mathrm{~h}$ at $6^{\circ} \mathrm{C}$, so as to form a film. Mica and initiator amounts are variables in this experiment. The formed chitosan $/ \mathrm{mica}$
TABLE 1: Code description of various copolymers.

\begin{tabular}{lc}
\hline Code & Description \\
\hline NM-967 & Modified mica \\
CS & Chitosan \\
$\mathrm{CS}_{0} \mathrm{M}_{n}$ & NM-967 blank test (without CS) \\
$\mathrm{CSM}_{n}$ & $n$ is percentage of mica in copolymer \\
\hline
\end{tabular}

copolymer is labeled $\mathrm{CSM}_{n}$ (where $n=1,2,4,6$, and 8 , the percentage of mica in the copolymer material) (Table 1).

2.3. Analysis and Inspection. FT-IR/ATR spectra of the finished fabrics were recorded with a Bio-Rad Digilab FTS-200 spectrometer using an MCT detector. A diamond crystal was used as the internal reflectance element. Single beam spectra were the result of 64 scans. The spectral resolution was $4 \mathrm{~cm}^{-1}$. The thermal resistance of samples was measured by a DuPont 2200 weight-loss analyzer. Samples (5-10 mg) were placed in alumina crucibles and tested over a temperature range of $30-600^{\circ} \mathrm{C}$, with a heating rate of $20^{\circ} \mathrm{C} / \mathrm{min}$ under a nitrogen flow of $20 \mathrm{cc} / \mathrm{min}$. After this step, their initial decomposition temperature (IDT) was obtained. A BrukerAXS D8 was used for X-ray powder diffraction (Parallel beam optics, Cu-target, scintillation counter, sampler changer with rotation). The samples were run with $40 \mathrm{kV}, 100 \mathrm{~mA}, 5-$ $50^{\circ}, 0.01^{\circ}$ stepwise, and $5 \mathrm{sec}$ counting time. Particle size distribution and the zeta potential of chitosan nanoparticles were determined using Zetasizer Nano-ZS90 (Malvern Instruments). The analysis was performed at a scattering angle of $90^{\circ}$ at a temperature of $25^{\circ} \mathrm{C}$ using samples diluted to different concentration with deionized distilled water. The surface morphologies of the films were observed with a JEOL Model JSM 5610 scanning electron microscopic. A gold coating was deposited on the samples to avoid charging the surface. A Jasco V-500 ultraviolet radiation analyzer was used for anti-UV light of the samples.

\section{Results and Discussions}

3.1. NM-967 Surface Microstructure. Figure 1 shows the SEM images of modified mica (NM-967) and $\mathrm{CSM}_{n}$ in a blank test. As shown in Figures 1(a) and 1(b), the microstructure of the modified mica powder is clearly particulate or lamellar. In Figures 1(c) and 1(d), the mica exhibits clear dendrites, lamella, and particles after the blank test. The dendrites are a sign of mica structures peeling off during the reaction, which facilitates processing and high barrier properties.

\subsection{Chitosan/Mica Copolymer Film}

3.2.1. FTIR. Figure 2 shows the FTIR spectra of various $\mathrm{CSM}_{n}$ copolymers. According to Paula Gomes et al. [20], the characteristic absorption peaks of chitosan are as follows: $3274 \mathrm{~cm}^{-1}, 2908 \mathrm{~cm}^{-1}, 1636 \mathrm{~cm}^{-1}, 1547 \mathrm{~cm}^{-1}, 1408 \mathrm{~cm}^{-1}$, $1367 \mathrm{~cm}^{-1}, 1260 \mathrm{~cm}^{-1}, 1070 \mathrm{~cm}^{-1}, 1026 \mathrm{~cm}^{-1}, 1152 \mathrm{~cm}^{-1}$, and $891 \mathrm{~cm}^{-1}$. The prominent peak at $3274 \mathrm{~cm}^{-1}$ is associated with an overlap between hydroxyl groups $(\mathrm{O}-\mathrm{H})$ and amino groups $(\mathrm{N}-\mathrm{H})$. The absorption peaks for $\mathrm{C}-\mathrm{H}$ are 


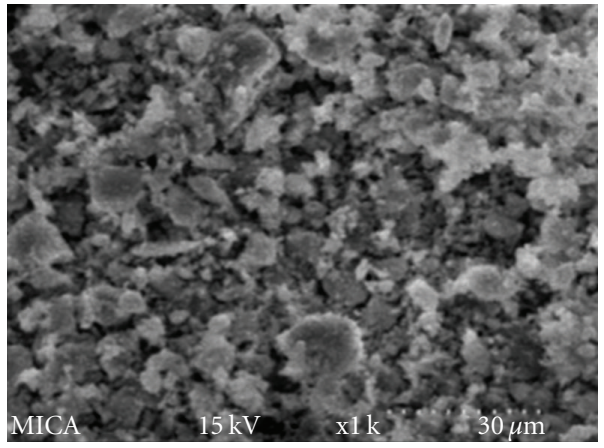

(a)

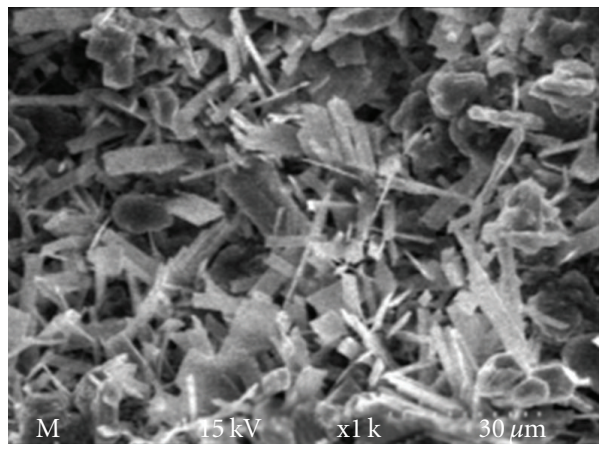

(c)

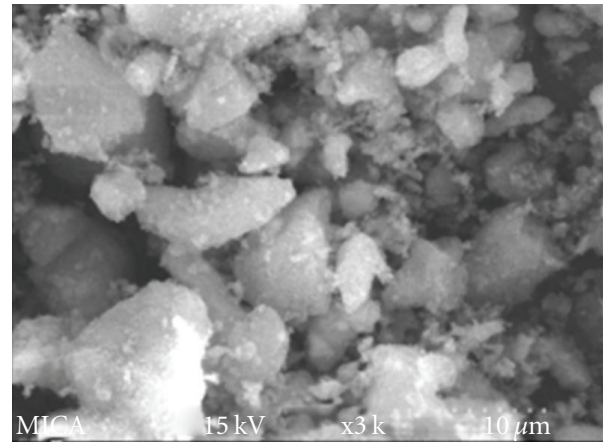

(b)

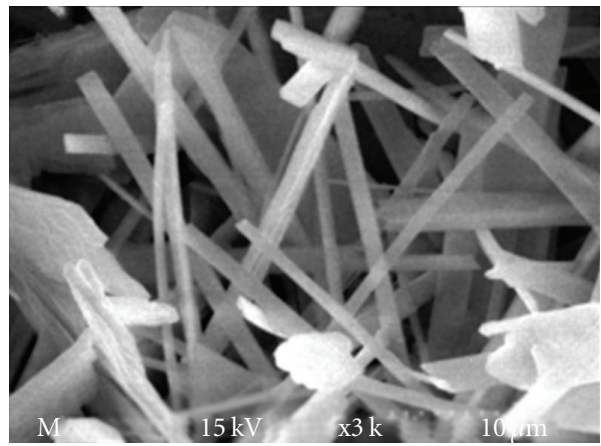

(d)

Figure 1: SEM images of modified mica (NM-967) and $\mathrm{CS}_{0} \mathrm{M}_{n}$ ((a-b) NM-967, (c-d) $\mathrm{CS}_{0} \mathrm{M}$, (a-c) $1.00 \mathrm{~K}$, (b-d) 3.00 K).

found at $2908 \mathrm{~cm}^{-1}$. Because the degree of deacetylation of chitosan is $85 \%$, there is still some chitin in the starting material. Therefore, several characteristic amide absorption peaks are present in the spectra $\left(\mathrm{C}=\mathrm{O}: 1636 \mathrm{~cm}^{-1}, \mathrm{~N}-\mathrm{H}\right.$ : $1547 \mathrm{~cm}^{-1}$, 3) C-N, N-H: $1408 \mathrm{~cm}^{-1}$; $-\mathrm{CH}_{3}: 1367 \mathrm{~cm}^{-1}$ ). Additionally, O-H absorption peak are found at $1260 \mathrm{~cm}^{-1}$, while distinct overlapping peaks at from $\mathrm{C}-\mathrm{O}-\mathrm{C}$ and $\mathrm{C}$ $\mathrm{NH}_{2}$ are apparent at $1070 \mathrm{~cm}^{-1}$ and $1026 \mathrm{~cm}^{-1}$, respectively. Finally, $\beta-(1,4)$ glycosidic bond absorption peaks are present at $1152 \mathrm{~cm}^{-1}$ and $891 \mathrm{~cm}^{-1}$.

In addition, as seen in Figure 2, the original -C-O-Cabsorption peak of CS at $107 \mathrm{~cm}^{-1}$ shifts to $1098 \mathrm{~cm}^{-1}$ and $1100 \mathrm{~cm}^{-1}$, forming an enhanced absorption peak at $1100 \mathrm{~cm}^{-1}$, in terms of $\mathrm{CSM}_{1}, \mathrm{CSM}_{4}$, and $\mathrm{CSM}_{8}$. This change is due to new $\mathrm{C}-\mathrm{O}$ and $\mathrm{C}-\mathrm{O}-\mathrm{C}$ bonds forming in during the copolymer material copolymerization and merge into one spectral zone. The absorption peak intensity decreases gradually as more mica is added because there is insufficient initiator to provide enough free radicals for all of the mica alkene bonds $(\mathrm{C}=\mathrm{C})$ to react and form $\mathrm{C}$ $\mathrm{O}-\mathrm{C}$. Hence, $\mathrm{CSM}_{8}$ has a small Si-O-C absorption peak at $1023 \mathrm{~cm}^{-1}$. Additionally, characteristic absorption peaks of amide structure in the $\mathrm{CSM}_{n}$ are located at $1634 \mathrm{~cm}^{-1}$, $1549 \mathrm{~cm}^{-1}$, and $1410 \mathrm{~cm}^{-1}$; these peaks intensify with the addition of mica. Moreover, the absorption peaks associated with $\beta$ - $(1,4)$ glycosidic bond at $1146 \mathrm{~cm}^{-1}$ and $897 \mathrm{~cm}^{-1}$ exhibit no significant change, indicating that the $\beta$-glycosidic bonds are not major active groups in reaction.

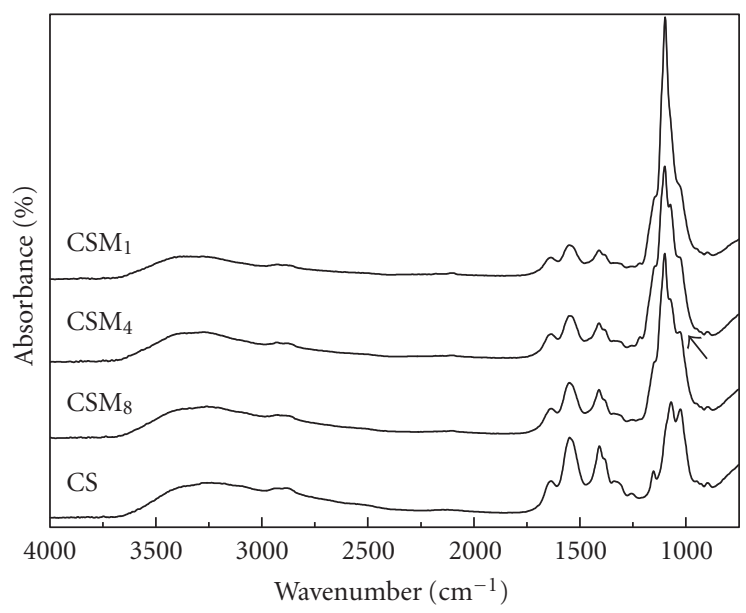

FIGURE 2: FTIR spectrum of CS and $\mathrm{CSM}_{n}$.

3.2.2. $X-R D$. Figure 3 shows the $\mathrm{X}-\mathrm{RD}$ spectra of various copolymers. According to Caiqin Qin et al. [21], CS has three significant diffraction peaks at $2 \theta$, angles of $11.05^{\circ}$, $17.92^{\circ}$, and $22.28^{\circ}$, which are attributed to chitosan's crystal structure (tendon hydrate polymorph). Taruta et al. [22] reported that mica has a single broad diffraction peak at $2 \theta$ of $25.70^{\circ}$ for its crystallized form. At $2 \theta=11.05^{\circ}$, a hydration crystallization peak, which is unique for chitosan, is present. 


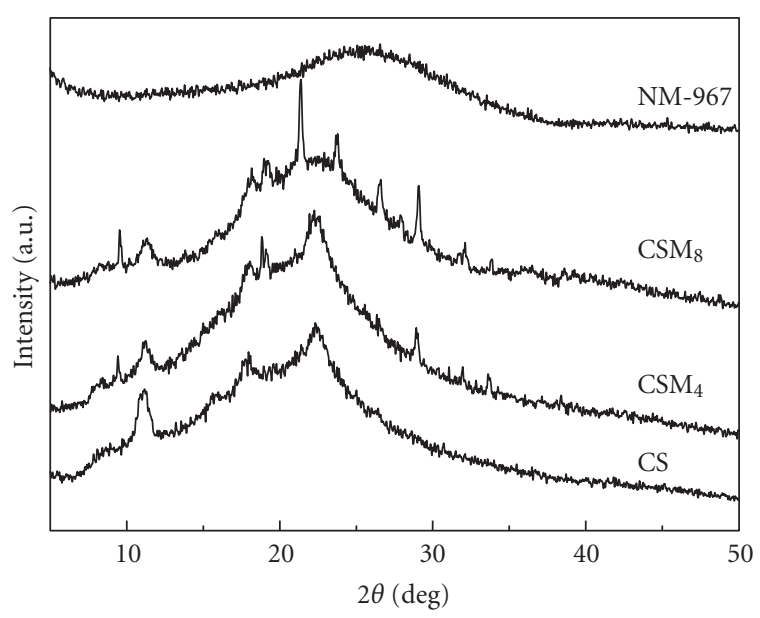

Figure 3: X-RD patterns of CS and $\mathrm{CSM}_{n}$.

Table 2: Particle Size CS and $\mathrm{CSM}_{n}$ copolymer emulsions.

\begin{tabular}{lcccccc}
\hline Products & $\mathrm{CS}$ & $\mathrm{CSM}_{1}$ & $\mathrm{CSM}_{2}$ & $\mathrm{CSM}_{4}$ & $\mathrm{CSM}_{6}$ & $\mathrm{CSM}_{8}$ \\
\hline Particle size $(\mathrm{nm})$ & 2013 & 817 & 651 & 781 & 947 & 1446 \\
\hline
\end{tabular}

As the mica content increases, diffraction peak turns weaker due to shielding by the layered structure of mica. Moreover, $\mathrm{CSM}_{4}$ and $\mathrm{CSM}_{8}$ have additional diffraction peaks at $2 \theta$ angles of $9.40^{\circ}, 18.95^{\circ}, 26.32^{\circ}, 29.03^{\circ}, 32.04^{\circ}$, and $33.81^{\circ}$. Based on the above, the added mica reacts with chitosan and leads to some changes in the crystal structure of chitosan.

3.2.3. Particle Size. Figure 4 and Table 2 show the particle size data of chitosan and various copolymer emulsions. Stockman et al. found that the stability of chitosan nanoparticles is related to hydrogen bonding between amino group $\left(-\mathrm{NH}_{2}\right)$, hydroxyl group $(-\mathrm{OH})$ of chitosan, and hydroxyl groups from water, and oxygen atoms of water. More hydrogen bonding induces aggregation more easily, resulting in larger particle size. Less hydrogen bonding enables particle dispersion and results in smaller particle size $[23,24]$. As compared with Figure 5, chitosan contains abundant hydroxyl groups and amino groups, which could easily form hydrogen bonds and cause aggregation. The average CS particle size is approximately $2000 \mathrm{~nm}$. After dehydrogenation and dehydration during copolymerization (following reaction mechanisms A and $\mathrm{B}$, resp.), the particle sizes of $\mathrm{CSM}_{n}(\mathrm{n}=1,2,4,6)$ get significantly smaller, ranging from 600-900 nm. However, the size of $\mathrm{CSM}_{8}$ particles is larger (about $1450 \mathrm{~nm}$ ) due to aggregation of excess mica.

3.2.4. SEM. Figure 5 shows the SEM images of chitosan and various copolymers. Film surface microstructures were examined under field emission scanning electron microscope at 5000x magnification. As seen in Figure 5(a), the microstructure of the pure chitosan film is smooth, without porosity, delamination, or insoluble particles. In Figures 5(b) and 5(c), both $\mathrm{CSM}_{4}$ and $\mathrm{CSM}_{8}$ have distinct dendritic surface microstructures. As mica is peeled off and dispersed

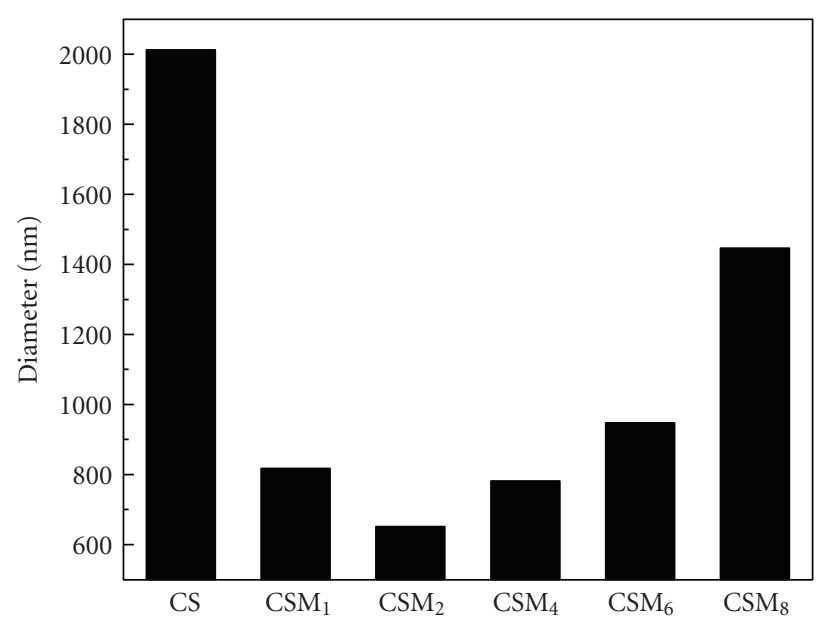

Figure 4: Particle size data of chitosan and various copolymer emulsions.

in chitosan films, unique layers of mica are observed (Figure 5(b)). In Figure 5(c), $\mathrm{CSM}_{8}$ mica aggregates are uneven in their distribution; hence, its barrier properties will be worse than $\mathrm{CSM}_{4}$, which corresponds to TGA graphs in Figure 6 .

3.2.5. TGA. Figure 6 and Table 3 are TGA graphs and thermal data of various copolymers, respectively. At $30^{\circ} \mathrm{C} \sim$ $100^{\circ} \mathrm{C}$, samples exhibit weight losses due to pure chitosan evaporation of moisture in the materials and the dehydration of crystalline water $(-\mathrm{OH})$ molecule within the interlayer of mica. As seen in Table 3, the pyrolysis temperatures of chitosan/mica copolymers are significantly higher than that of pure chitosan, indicating that the addition of mica leads to changes in the chitosan structure. The thermal degradation of the chitosan occurred in the temperature range of $200^{\circ} \mathrm{C} \sim$ $450^{\circ} \mathrm{C}$. The weight loss in the temperatures of the curves of various copolymers might be associated with the thermal degradation of the amine or the residual $N$-acetyal on chitosan. Intercalation of organic species in layered inorganic matrix is well known to alter their thermal behavior. The unique mica lamellar structures enable the high barrier properties of these copolymer materials, resulting in improved thermal stability. The rate of mass loss decreases as the mica content is increased; the pyrolysis temperature and mass of the remaining residue increase as well. However, the thermal stability of $\mathrm{CSM}_{8}$ dissipates as more mica is added, resulting in uneven dispersion. Also, hydroxyl groups in mica fail to dehydrate completely during the reaction, it is attributed to adsorbed water as in the cellulose mica nanocopolymer.

3.2.6. Anti-UV. Figure 7 shows the UV graphs of chitosan and various copolymer emulsions. After adding mica, the UV light absorption increases as the unique lamellar structure of mica leads to a crystal polarization effect and a light interference effect due to a layer of crystallized water. Furthermore, Si particles scatter and block the light, making copolymer material more resistant to UV wavelengths. Based 


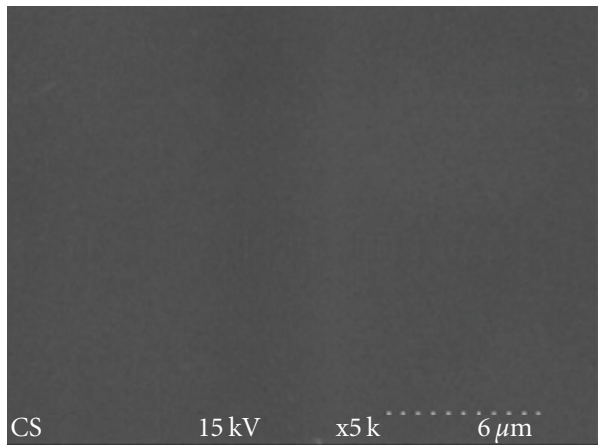

(a)

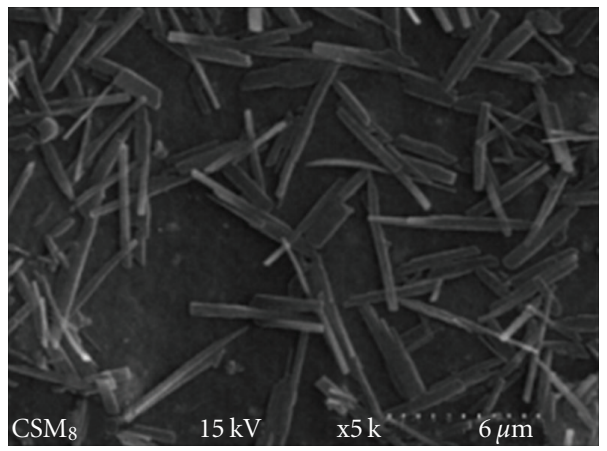

(c)

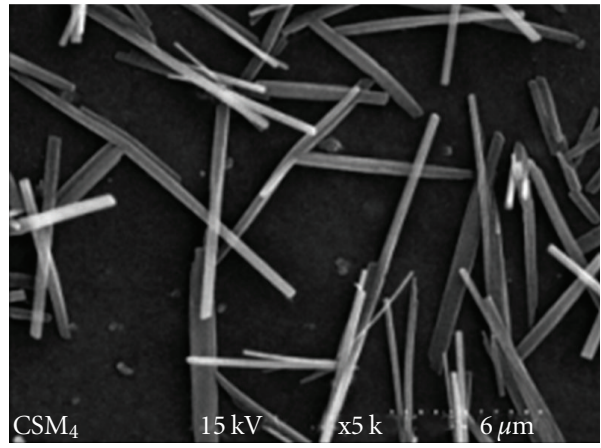

(b)

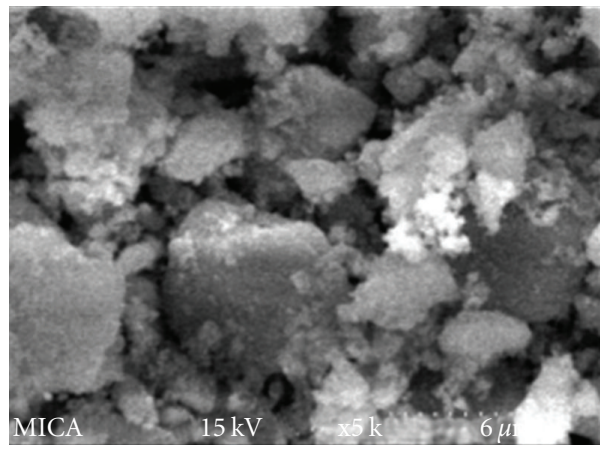

(d)

Figure 5: SEM images of chitosan and $\mathrm{CSM}_{n}$. ((a) CS, (b) $\mathrm{CSM}_{4}$, (c) $\mathrm{CSM}_{8}$, (d) NM-967).

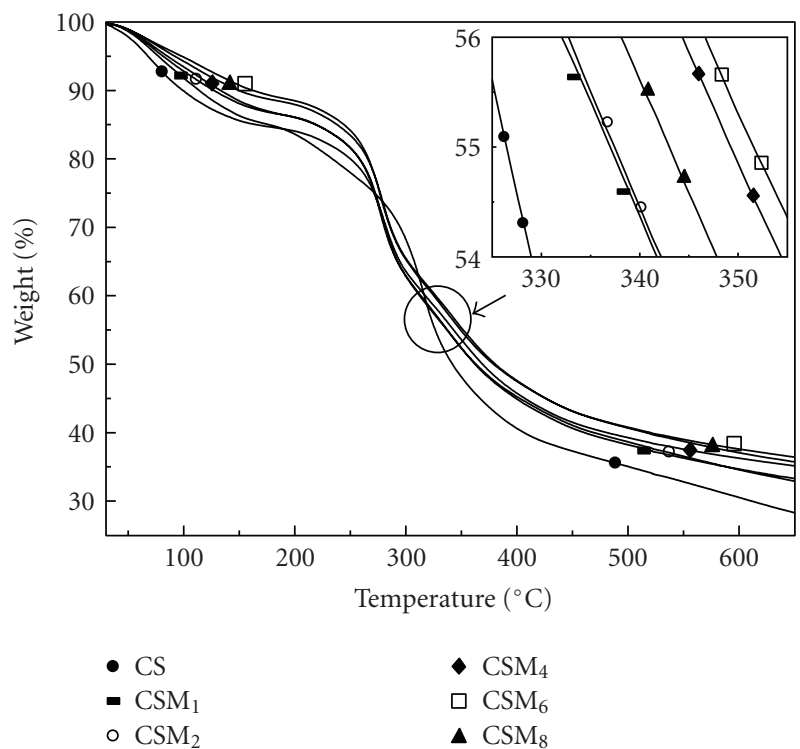

FIgURE 6: TGA of Chitosan and $\mathrm{CSM}_{n}$.

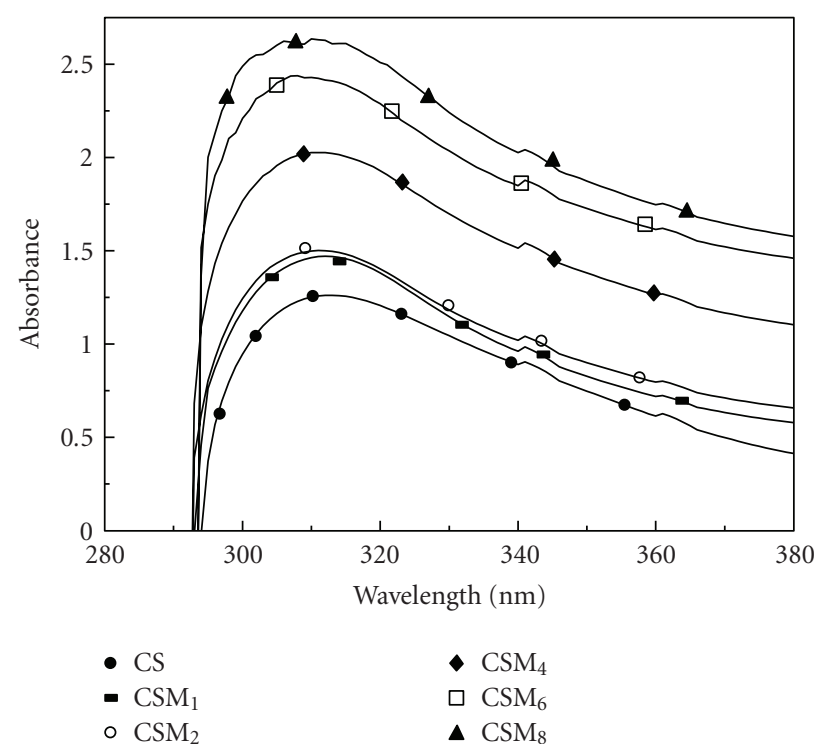

Figure 7: UV absorption spectrum of CS and $\mathrm{CSM}_{n}$. on these results, the addition of mica imparts copolymer with high barrier properties, resulted in enhanced UV light resistance. These properties become more significant with increasing mica content.

\section{Conclusions}

This study used modified mica and chitosan to produce a copolymer and formulated various copolymer emulsions 
TABLe 3: Thermal properties of copolymers.

\begin{tabular}{lcccc}
\hline Samples & \multicolumn{4}{c}{ Thermal properties } \\
& $T_{20}\left({ }^{\circ} \mathrm{C}\right)$ & $\begin{array}{c}\text { Decomposition } \\
\text { rate }\left(\% /{ }^{\circ} \mathrm{C}\right)\end{array}$ & $T_{45}\left({ }^{\circ} \mathrm{C}\right)$ & $\begin{array}{c}\text { Residual } \\
\mathrm{mass}^{1}(\%)\end{array}$ \\
\hline $\mathrm{CSM}_{8}$ & 242.18 & -0.399 & 341.22 & 35.14 \\
$\mathrm{CSM}_{6}$ & 265.53 & -0.445 & 351.58 & 36.42 \\
$\mathrm{CSM}_{4}$ & 266.50 & -0.456 & 349.27 & 35.73 \\
$\mathrm{CSM}_{2}$ & 258.14 & -0.461 & 337.44 & 33.30 \\
$\mathrm{CSM}_{1}$ & 258.41 & -0.463 & 336.98 & 32.89 \\
$\mathrm{CS}$ & 233.77 & -0.487 & 326.46 & 28.31 \\
\hline
\end{tabular}

at $650^{\circ} \mathrm{C}$.

into film. The results found that the addition of mica affected both film surface microstructure and crystal structure. However, when the added amount exceeds $8 \%$, microstructure aggregates may appear. The copolymer particle size resulting from the emulsion is smaller than that of chitosan; the size does not increase when excess chitosan is added. Compared with chitosan, the copolymer film has better thermal stability and UV absorption, which becomes more prominent as the amount of added mica increases. However, when the amount of mica is greater than $8 \%$, the thermal stability decreases.

\section{References}

[1] Y. C. Chung and C. Y. Chen, "Antibacterial characteristics and activity of acid-soluble chitosan," Bioresource Technology, vol. 99, no. 8, pp. 2806-2814, 2008.

[2] Y. T. Shieh and Y. F. Yang, "Significant improvements in mechanical property and water stability of chitosan by carbon nanotubes," European Polymer Journal, vol. 42, no. 12, pp. 3162-3170, 2006.

[3] G. M. Spinks, S. R. Shin, G. G. Wallace, P. G. Whitten, S. I. Kim, and S. J. Kim, "Mechanical properties of chitosan/CNT microfibers obtained with improved dispersion," Sensors and Actuators B, vol. 115, no. 2, pp. 678-684, 2006.

[4] H. Caner, E. Yilmaz, and O. Yilmaz, "Synthesis, characterization and antibacterial activity of poly(N-vinylimidazole) grafted chitosan," Carbohydrate Polymers, vol. 69, no. 2, pp. 318-325, 2007.

[5] M. N. V. Ravi Kumar, "A review of chitin and chitosan applications," Reactive and Functional Polymers, vol. 46, no. 1, pp. 1-27, 2000.

[6] M. N. V. Ravi Kumar, R. A. A. Muzzarelli, C. Muzzarelli, H. Sashiwa, and A. J. Domb, "Chitosan chemistry and pharmaceutical perspectives," Chemical Reviews, vol. 104, no. 12, pp. 6017-6084, 2004.

[7] O. Felt, P. Buri, and R. Gurny, "Chitosan: a unique polysaccharide for drug delivery," Drug Development and Industrial Pharmacy, vol. 24, no. 11, pp. 979-993, 1998.

[8] S. Hirano, H. Seino, I. Akiyama, and I. Nonaka, "Biocompatibility of chitosan by oral and intravenous administration," Polymer Engineering Science, vol. 59, pp. 897-901, 1989.

[9] R. A. A. Muzzarelli, "Human enzymatic activities related to the therapeutic administration of chitin derivatives," Cellular and Molecular Life Sciences, vol. 53, no. 2, pp. 131-140, 1997.

[10] P. C. Bersch, B. Nies, and A. Liebendorfer, "Evaluation of the biological properties of different wound dressing materials," Journal of Material Science, Materials in Medicine, vol. 6, pp. 231-240, 1995.
[11] R. Jayakumar, M. Prabaharan, R. L. Reis, and J. F. Mano, "Graft copolymerized chitosan-present status and applications," Carbohydrate Polymers, vol. 62, no. 2, pp. 142-158, 2005.

[12] H. S. Blair, J. Guthrie, T. K. Law, and P. Turkington, "Chitosan and modified chitosan membranes I. preparation and characterization," Journal of Applied Polymer Science, vol. 33, no. 2, pp. 641-656, 1987.

[13] M. Terbojevich, A. Cosani, and R. A. A. Muzzarelli, "Molecular parameters of chitosans depolymerized with the aid of papain," Carbohydrate Polymers, vol. 29, no. 1, pp. 63-68, 1996.

[14] Z. Rong and L. Zhu, "Preparation and application of graft copolymer of 2,3- epoxypropyltrimethylammonium chloride onto chitosan," Paper Chemicals, vol. 19, no. 2, pp. 19-22, 2007.

[15] M. J. Zohuriaan, "Advances in chitin and chitosan modification through graft copolymerization: a comprehensive review," Iranian Polymer Journal, vol. 14, no. 3, pp. 235-265, 2005.

[16] G. D. Liang, J. T. Xu, Z. Q. Fan, S. M. Mai, and A. J. Ryan, "Effect of substrate surface on dewetting behavior and chain orientation of semicrystalline block copolymer thin films," Journal of Physical Chemistry B, vol. 110, no. 48, pp. 2438424389, 2006.

[17] J. Kumaki, T. Kawauchi, and E. Yashima, "Two-dimensional folded chain crystals of a synthetic polymer in a langmuirblodgett film," Journal of the American Chemical Society, vol. 127, no. 16, pp. 5788-5789, 2005.

[18] H. Heinz, H. J. Castelijns, and U. W. Suter, "Structure and phase transitions of alkyl chains on mica," Journal of the American Chemical Society, vol. 125, no. 31, pp. 9500-9510, 2003.

[19] G. Jiang, T. Wang, J. Zhang, and X. Shen, "Effect of mica filler on anti-UV aging of polypropylene composite," China Synthetic Resin and Plastics, vol. 25, no. 1, pp. 35-39, 2008.

[20] P. Gomes, C. A.R. Gomes, M. K.S. Batista, L. F. Pinto, and P. A.P. Silva, "Synthesis, structural characterization and properties of water-soluble N-( $\gamma$-propanoyl-amino acid)-chitosans," Carbohydrate Polymers, vol. 71, no. 1, pp. 54-65, 2008.

[21] C. Qin, H. Li, Q. Xiao, Y. Liu, J. Zhu, and Y. Du, "Watersolubility of chitosan and its antimicrobial activity," Carbohydrate Polymers, vol. 63, no. 3, pp. 367-374, 2006.

[22] S. Taruta, R. Fujisawa, and K. Kitajima, "Preparation and mechanical properties of machinable alumina/mica composites," Journal of the European Ceramic Society, vol. 26, no. 9, pp. 1687-1693, 2006.

[23] P. A. Stockman, R. E. Bumgarner, S. Suzuki, and G. A. Blake, "Microwave and tunable far-infrared laser spectroscopy of the ammonia-water dimer," The Journal of Chemical Physics, vol. 96, no. 4, pp. 2496-2510, 1992.

[24] G. A. Yeo and T. A. Ford, "Ab initio molecular orbital calculations of the infrared spectra of hydrogen bonded complexes of water, ammonia, and hydroxylamine," Canadian Journal of Chemistry, vol. 69, no. 4, pp. 632-637, 1991. 

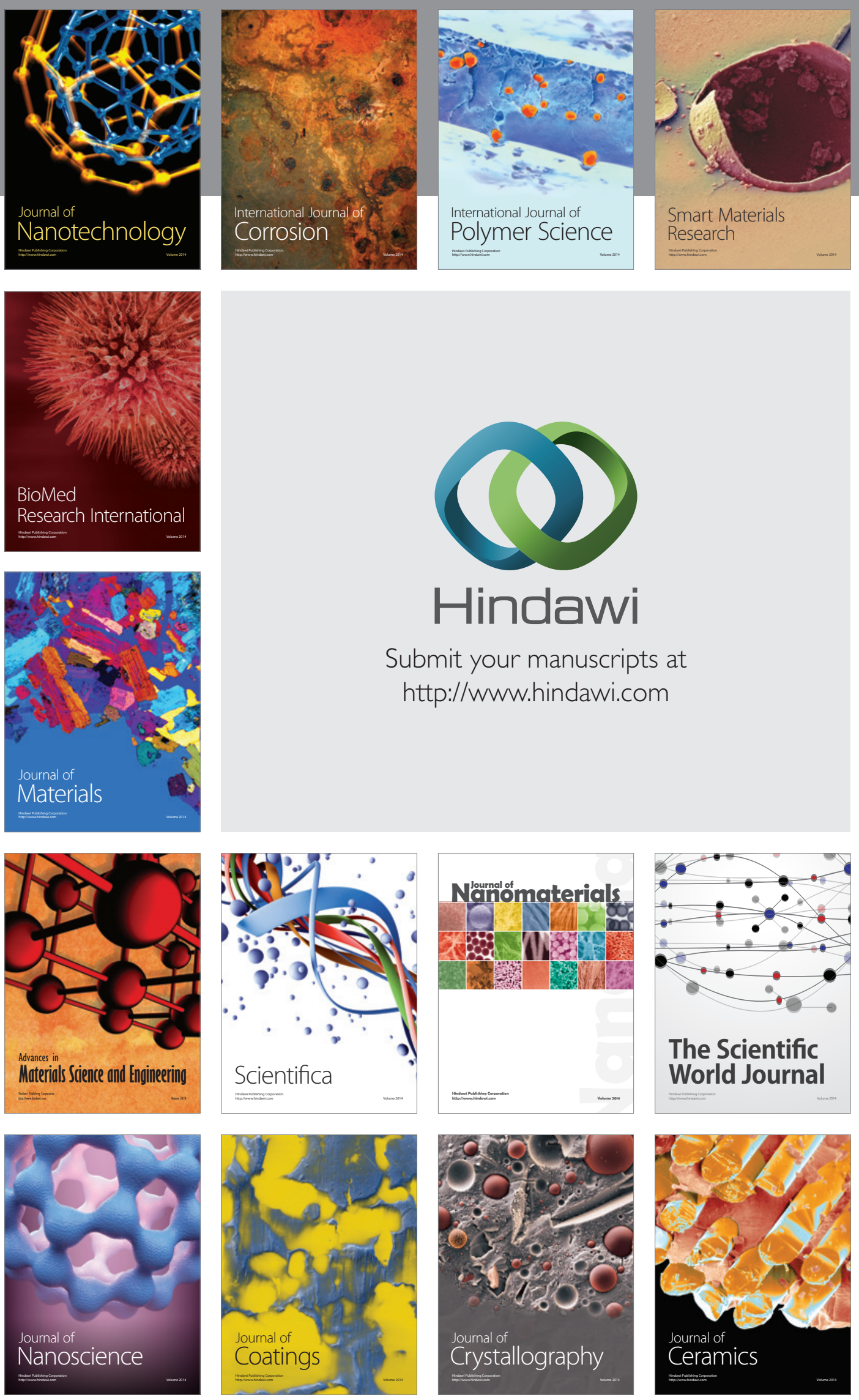

The Scientific World Journal

Submit your manuscripts at

http://www.hindawi.com

\section{World Journal}

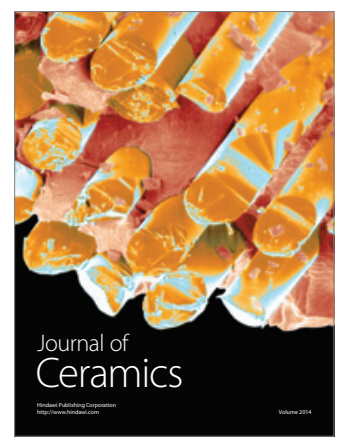

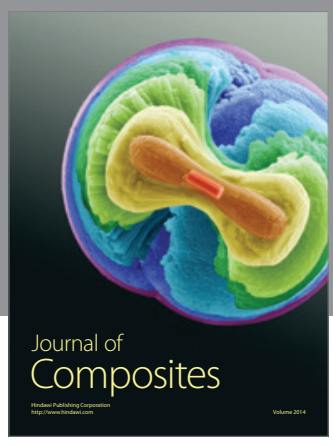
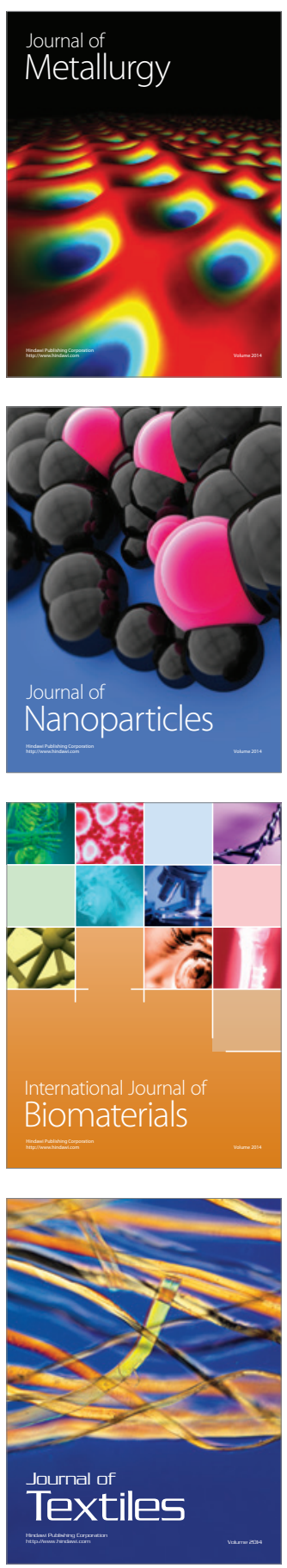